\title{
Téoros
}

Revue de recherche en tourisme

\section{Le marché des congrès à Montréal}

\section{Marie-Andrée Camirand}

Volume 10, numéro 3, novembre 1991

Destination Montréal : d'hier à demain

URI : https://id.erudit.org/iderudit/1079209ar

DOI : https://doi.org/10.7202/1079209ar

Aller au sommaire du numéro

Éditeur(s)

Université du Québec à Montréal

ISSN

0712-8657 (imprimé)

1923-2705 (numérique)

Découvrir la revue

Citer cet article

Camirand, M.-A. (1991). Le marché des congrès à Montréal. Téoros, 10(3), 19-24. https://doi.org/10.7202/1079209ar d'utilisation que vous pouvez consulter en ligne.

https://apropos.erudit.org/fr/usagers/politique-dutilisation/ 


\section{Le marché des}

Marie-Andrée Camirand" congrès à Montréal

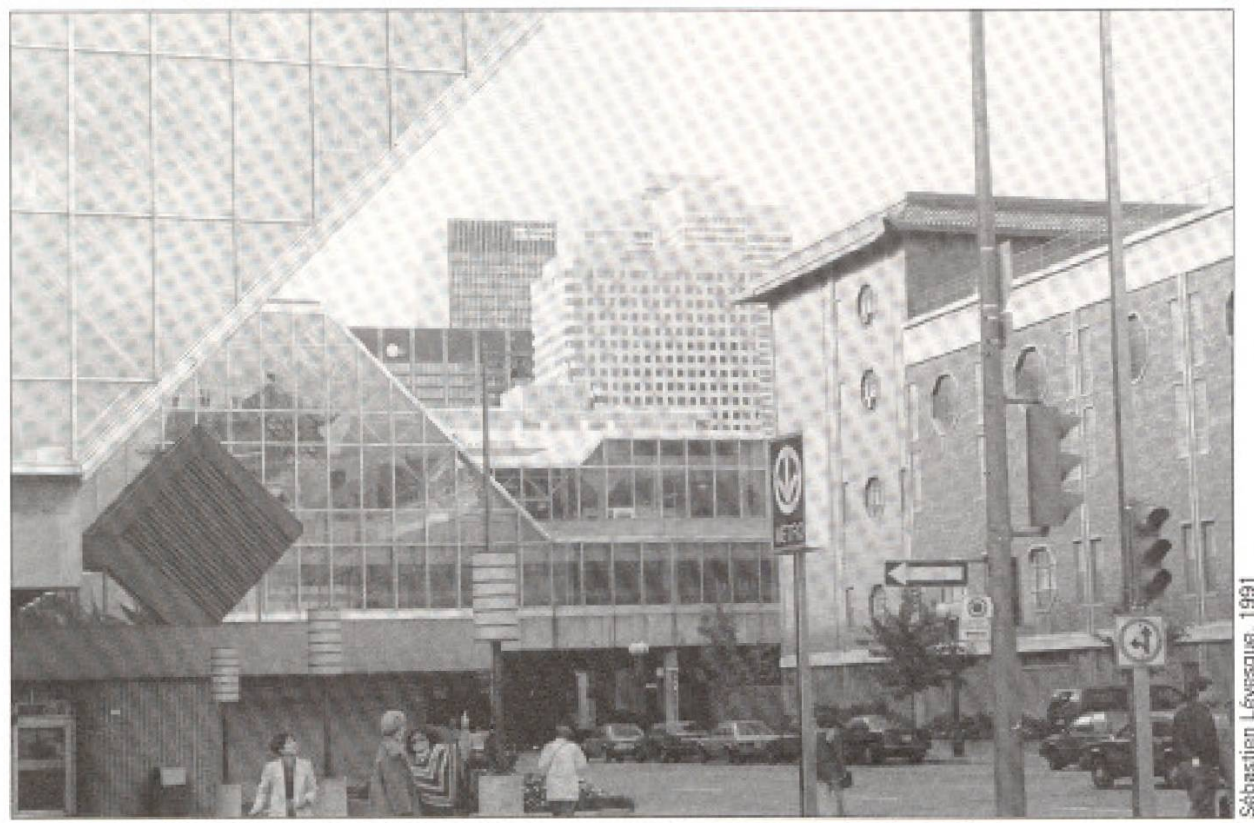

La demande prewsible est telle que la Palais des Congrés de Montréal ne suffira bientöt plus à la tiche.

\section{Introduction}

Lorsqu"on évoque le terme congrès, on pense spontanément à un groupe de personnes qui, pour des raisons diverses (politiques, sociales, économiques, professionnelles, religieuses, etc.) se rassemblent afin de confronter leurs idées, partager différents points de vue, échanger de l'information et ainsi faire progresser leurs recherches professionnelles, discuter de différents problèmes inhérents à leur organisation ou pour définir de nouveaux objectifs.

Globalement, l'organisation de congrès demeure une activité complexe qui exige l'implication de plusieurs intervenants oeuvrant dans différents domaines et qui permet des retombées économiques souvent impressionnantes.

On ne peut certainement pas comparer le nombre de réunions d'entreprises au nombre de congrès organisés par les associa-

Madame Mariéandrée Camirand est directrice-adjointe au developpement al'Office des congrtes et du 1ourisme du Grand Montréal (Q.C.T.G.M.). tions car de toute évidence, de par leur fonctionnement, les entreprises se réunissent beaucoup plus souvent que les associations. Toutefois, pour les fins de cet article, nous nous attardons spécifiquement aux congrès d'associations qui constituent la plus importantepart du marché des réunions tant en terme de volume des participants qu'en terme de retombées économiques inhérentes à cette activité.

Pour brosser le plus précisément possible le tableau des congrès à Montréal, on doit d'abord le définirpar catégoriesde marchés: américain, international, canadien et québécois.

\section{Marché des congrès américains}

Comme nous le mentionnions précédemment, il est reconnu que les congrès d'associations constituent la plus importante part du marché des réunions. D'ailleurs, sur la scène américaine, les retombées économiques résultant de cette activité le confirment. En effet, on remarque que même si les congrès d'associations ne constituent que $1 \%$ du total des réunions, ils génèrent à eux seuls la majeure partie des dépenses effectuées par les abonnés du Meetings and Conventions (voir tableau 1).

Ces derniers, rappelons-le, constituent la majorité des décideurs et organisateurs des réunions de tous genres aux États-Unis.

Comme les dépenses liées à ce marché sont en pleine croissance (+ de $37 \%$ entre 1987 et 1989), le nombre de délégués présents aux congrès d'associations tend également à augmenter.

La délégation moyenne des associations américaines est passée de 848 en 1987 à 1078 en 1989 , soit une augmentation de $27 \%$. De plus, chaque association dépense en moyenne $121400,00 \$$ par année pour organiser son congrès annuel. Enfin, on estime à environ 989 \$ US les dépenses d'un délégué participant à un congrès de son association.

Lorsqu'une association américaine décide de tenir un congrès à l'étranger, le Canada demeure bon premier. Selon l'Association Meeting Trends, des 8000 associations interviewées, $13 \%$ d'entre elles ont tenu leur congrès à l'extérieur des USA entre 19851988. La majorité de ces congrès ont eu lieu au Canada (53\%).

On note également une tendance à tenir conjointement une exposition et un congrès.

\section{Tendances du marché américain des congrès au Canada et à Montréal}

Le marché américain des congrès d'associations est un bassin de clientèle des plus intéressants pour Montréal. La majorité des sièges sociaux des associations américaines sont localisés dans la partie est des États-Unis soit dans les États près du Québec; la plus grande proportion se retrouve à New York, en Illinois, à Washington D.C., en Virginie et dans le Maryland(i) 
TABLEAU 1

Relation entre le nombre de réunions de tous genres

et les dépenses qu'elles entrainent

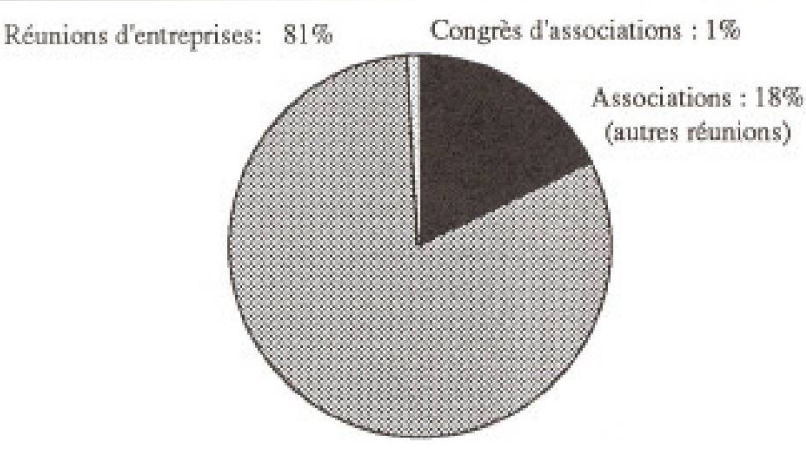

Réunions de tous genres (en nombre de réunions)

Total: 1066000

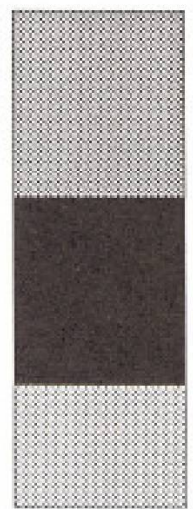

Congrès d'associations : $15 \$ 37.9 \%$

Associations (autres tétunions): $14.9 \$ 37.6 \%$

Réunions d'entreprises : $9.7 \$ 24.5 \%$

Defpenses (en milliards)

Total: $39.6 \$$

Source: Meetings Market, p. 4.

On constate Également que $34 \%$ de ces associations préferent tenir leurs congrès dans l'est américain.

\section{Au Canada}

Pour les Américains, le Canada est un pays attirant parce que ses villes ont un caractere différent: elles sont belles, propres et disposent d'installations adéquates toutenétant proches et pourvues de toutes les caractéristiques modernes des villes nord-américaines. En fait, selon Hutchinson-Reid, ale Canada est différent, exotique, stimulant, chic sans être trop differento. Metropoll III place Montréal au 22 e rang parmi les 50 destinations-congrès nord-américainesque choisissent les associations americaines pour tenir leurs congrès.

Au Canada, la ville de Montréal se classe troisieme derriêre Toronto et Vancouver. Calgary, Halifax et Québec occupent les 4 e, 5 e et 6 e place.

On remarque egalement que le choix du lieu pour la plupart des rétunions d'affaires (réunions de tous genres et congres $\mathrm{d}^{\dagger}$ associations confondus) se fait souvent en fonction de l'endroit où se trouvent les filiales de l'organisation. Lorsqu'il y a flexibilite, comme c'est le cas pour plusieurs associations (qui cherchent avant tout a donner satisfaction à leurs membres) , on choisit presque toujours des centres ur- bains notamment Toronto, Montréal et Vancouver ${ }^{22}$.

Lorsque le choix se porte sur d'autres villes canadiennes, c'est en raison de liens existants entre les associations et les filiales sises dans les municipalites de moindre importance.

\section{À Montréal}

Dans le prochain chapitre, on considère d'une part les infrastructures et les commodités techniques qu'offre Montréal comme destination de congrès et d'autre part, on mesure l'intérêt touristique que la région suscite (son mode de vie, son confort, la qualité de ses équipements, etc.) chez les organisateurs de congres.

En ce qui concerne la perception qu'ont les organisateurs de congres américains du Palais des Congress de Montréal (PCM), l'étude Metropoll III révèle que Montréal ne se place jamais parmi les 25 premiers choix de destination (voir tableau 2).

De plus, le classement des caractéristiques propres aux Centres de Congrès et qui a servi de base de comparaison entre les différentes destinations de congrès nordaméricaines, róvèle une performance du PCM en-deça de la moyenne exprimée par l'indice 100 .
Comme destination-voyage, il en va autrement. Les organisateurs de congrès ont une toute autre perception de Montréal comme l'indique le tableau 3, le caractère unique, la qualitéde sa vie nocturne et la qualité de ses restawrants se retrouvent bien au-dessus de la moyenne lorsqu'on fait la comparaison avec les autres destinations-congres nordaméricaines.

Comparativement aux 50 autres destinations, Montréal est considéréce comme étant une ville dont les coûts pour s'y rendre sont relativement peu élevés. Elle possède un taux de criminalité relativement bas. C'est une ville attrayante, propre qui offre des possibilités préetpost congrèsintéressantes. Toutefois, Montréal demeure très compétitive aux plans de la popularité, de l'intérêt de ses régions, du sigh seeing, du magasinage et de son transport local.

Toujours selon Metropoll III, Montréal n'a reçu que peu de considération en ce qui concerne les items, service aérien adequat, bonne publicite, endroit relaxant, citoyens amicaux, bonne destination familiale et bon climat. Montréal est de plus considérée comme une ville ni trop chaleureuse ni trop amicale (voir tableau 3).

Quantà la perception que révèle Metropoll III du Palais des congrès, nous croyons que celle-ci a été corrigée, tenant compte de la décision du Palais d'entreprendre des démarches plus agressives sur le marché des 
associations américaines en ouvrant, entre autres, un bureaude représentation à Washington.

Le vent actuel de récession economique $\mathrm{n}$ 'aidant pas, les associations américaines sont moins portées à sortir des États-Unis pour tenir leurs congres. De plus, les états du Sud ont connu ces derniêres années une augmentation spectaculaire de leur parc hôtelier et de leurs centres de congrès. Ces hôtels en nombre trop élevé pour la demande réelle offrent leurs chambres à des prix parfois dếrisoires, tentant ainsi de S"accaparer une clientèle massive de congressistes pour combattre la forte compétitivité quil règne dans cette région et augmenter leur taux d'occupation.

Cette compétition n'est donc pas facile pour le Québec et le Canada surtout lorsque le prix des chambres que l'on offre ici ne peut que difficilement concurrencer celui des nouveaux équipements hôteliers américains.

\section{Tendances du marché des congrès internationaux}

Sur le plan international, Montréal en tant que destination-congrès occupe une place de premier choix. Cette année encore et ce, depuis l'ouverture du Palais des congrès, 1'Union des associations internationales (UAI) a classé Montréal au troisième rang des villes nord-américaines après New York et Washington et au premier rang des villes canadiennes pour la tenue de congrès internationaux (voir tableau 4).

Montréal est reconnue partout comme une ville possédant une expertise au niveau de l'accueil des congrès d'associations a caractère scientifique et notamment dans le domaine médical. En effet, $35 \%$ des congress scientifiques proviennent des sciences de la médecine tandis que $20 \%$ proviennent des domaines de la science pure et de la techno-ếnergie.

\section{Vue d'ensemble}

Depuis quelques années, le marché mondial des congrès internationaux a connu une montéc assez spectaculaire. Le rapport statistique de l'UAI de 1989 explique cette tendance en soulignant l'organisation de quelques grands congrès scientifiques (triennaux, quadriennaux) qui devaient en-

TABLFAU 2

Perception des décideurs et organisateurs de congrès du Palais des congrès de Montréal

Support de la communanté

Audio-visurel

Facile diacces

Proximité des hôtels

Nombre suffisant de chambres dhotel

Beau Centre de congres

Salle de réunions suffisantes

Nombre suffisant de places assises

Qualité de la nourriture

Bonnes installations pour banquets

Centre de Congres modeme

Serviabilité du persomnel

Competence de la direction

Aeroports (proximite)

Installations adéquates pour expositions

Amangements des expositions

Arrangements et services (prix)

Espaces suffisants pour expositions

Prix de location

Disponibilite

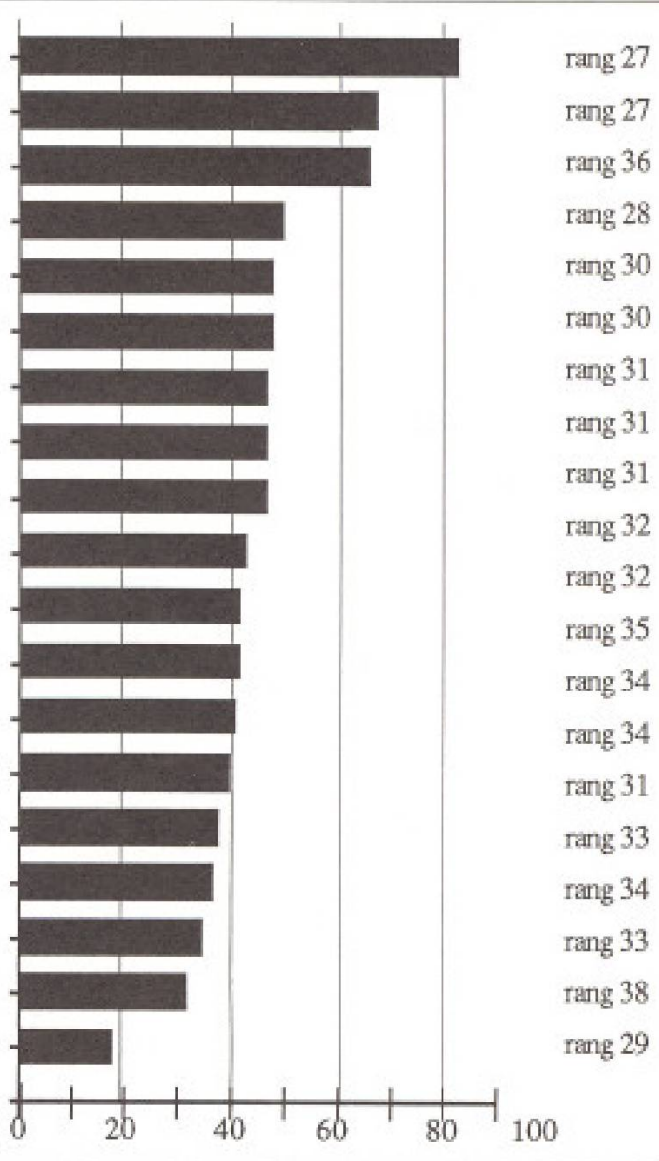

Source: Metropoll III, Economics Research Associates, volume II, 1990.

gendrer un grand nombre de réunions satellives ${ }^{(3)}$.

En 1989, le marché s'est stabilisé. Cependant, on note que c'est l'Amérique du Nord qui a enregistré la plus forte augmentation durant cette période, $+0,96 \%$.

Toutefois, globalement surce marché, c'est le continent européen qui demeute le chef de file, avec Paris en tête.

Sur le continent nord-américain, Washington et New York précèdent Montréal qui occupe bel et bien la troisième place, se retrouvant devant Toronto, San Francisco et Ottawa.

La concurrence entre Montréal et les autres villes canadiennes ne semble pas effrayer, outre mesure, les principaux solliciteurs de congrès internationaux. En 1990, Montráal occupe la troisième place parmi les destinations nord-américaines et a accueilli sept congress d'envergure internationale de plus que Toronto. N'oublions pas que cette dernière est davantage reconnue pour son expertise sur le marché des associations américaines.

Vancouver de son côté ne possède ni les infrastructures suffisantes (nombre de chambres d'hôtel, espaces disponibles, etc.) ni l'expertise pour concurrencer Montréal sur ce marché.

Les statistiques de l'UAI démontrent qu'il y a une majorité de réunions ayant moins de 1000 participants. Toutefois, ce sont les réunions de 100 à 500 participants qui demeurent les plus importantes $(62 \%)$ et ensuite, celles de 501 à $1000(13,60 \%)$. 
De plus, au niveau des expositions conjointes aux congrès, la tendance ne semble pas avoirévoluéet se situe toujoursà $10,5 \%$.

De façon générale, le marché des congrès internationaux est en plein essor: Montréal doit travailler constamment pour se maintenir dans la course et garder sa troisième position.

\section{Bref survol du marché des congrès canadiens}

Les données concernant le marché canadien sont beaucoup moins déttaillées. Toutefois, nous savons que les associations canadiennes ont, de façon générale, une bonne connaissance de Montréal comme destination-congrès. De plus, elles possèdent, la plupart du temps, un bureau chef dans une des villes d'importance au Canada. Ainsi, il est facile de les solliciter et de rejoindre les personnes responsables de l'organisation de congrês.

Enfin, les associations canadiennes exercent une rotation aux cinq ans environ, c'est-ầ-dire qu'elles reviennent à Montrâal tenir leur congrès après avoir visité d'autres villes canadiennes durant cette période.

Bien que quasiment acquis, ce marché ne devrait pas être négligé au Québec et à Montréal; il arrive trop souvent qu'on annule une réservation canadienne au profit d'une association américaine ou internationale.

\section{Quelques données du marché des congrès québécois}

La majorité des quelques 3000 associations duQuébec $(71 \%)$ ont une envergure québécoise e'est-à-dire que les membres de ces associations proviennentexclusivement du Québec. On note également que plus des deux tiers ont leur siège social dans la région immédiate de Montréal alors que $20 \%$ situent leur centre de décision dans le Québec métropolitain.

Ces associations tiennent, â $90 \%$, leur congrès (durée moyenne de deux ou trois jours, environ 100 participants) au Québec et surtout à Montréal.

Les associations et les clubs à caractère communautaire, social et culturel et les autres associations professionnelles con-

TABLEAU 3

Perception de Montréal comme destination congrès en comparaison avec 50 autres destinations nord-américaines

Peu dispendieux pour sy rendre

Unique/Différent

Vie nocture

Bons restaurants:

Possibilités préfost congrès

Ville altrayante

Faible criminalitéjsócurité

Magasinage

Sight seeing

Transport local

Endroit populaire

Coûis c̉levés (Hôtels et restaurants)

Region intéressante

Coutts moyens (Hôtels et restaurants)

Ciloyens amicaux

Bonte destination farmiliale

Endroit relaxant

Service aérien adéquat.

Faibles coùts (Hôtels et restuurants)

Bonne publicité

Bon climat

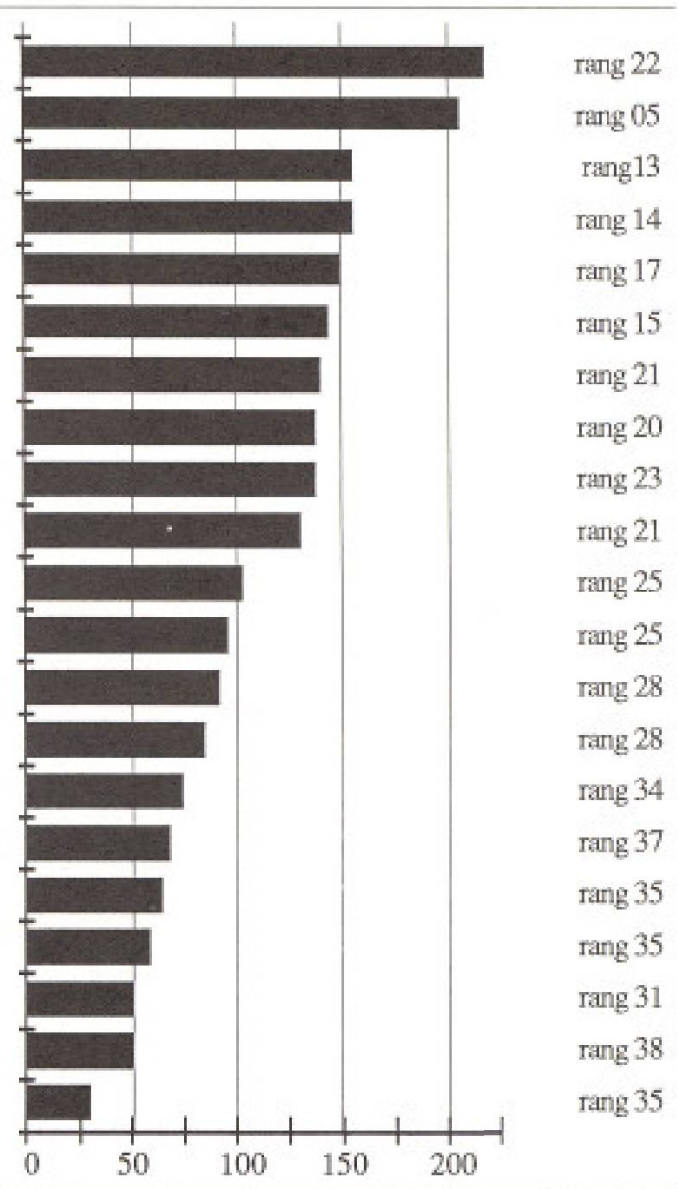

Source: Metropoll III, Economics Research Associates, volume II, 1990.

stituent la majorité des associations qui tiennent des congrès. Les autres étant les partis politiques, les syndicats ouvriers et les associations commerciales.

La plupart du temps, les congrès ont lieu dans les établissements hôteliers. Enfin, on remarque que de plus en plus de congrès d'associations se tiennent conjointement avec des expositions ( $39 \%$ en 1986$)^{4}$.

\section{Mise en marché}

À Montréal, la mise en marché des congrès implique une série d'acteurs allant du petit hôtelier aux vendeurs chevronnés du Palais des congrès de Montréal et de l'Office des congrès et du tourisme du Grand Montréal, sans oublier bien sûr le travail de prospection du ministère du Tourisme du Quếbec.
On note également que chacun des marchés possède ses spécialistes qui par différentes actions (sollicitation postale, visite sur le terrain, ambassadeurs, voyages de familiarisation, etc.) cherchent à attirer le plus grand nombre de congrès à Montréal.

Il faut noter que le Palais des congrès de Montréal a acquis une compétence enviable sur le marché international des congrès d'envergure. Les hôtels sollicitent êgalement les congrès internationaux mais ceux de moindre importance numérique. La troisième place en Amérique du Nord qu'occupe Montréal sur ce marché spécifique en dit long sur le travail de ces intervenants.

La sollicitation de congrès d'envergure qui s'effectue par le Palais des congrès exige une organisation efficace; il faut tetre rapide, 
TABLEAU 4

Principales villes ayant reçu des réunions d'organisations internationales et des réunions nationales avec participation internationale

\begin{tabular}{lll}
\hline Rang & Villes & $\begin{array}{l}\text { Nombre } \\
\text { de réunions }\end{array}$ \\
\hline 1 & Paris & 361 \\
2 & Londres & 268 \\
3 & Bruxelles & 194 \\
4 & Vienne & 177 \\
5 & Genève & 166 \\
6 & Berlin Ouest/Est & 166 \\
7 & Madrid & 161 \\
8 & Singapour & 138 \\
9 & Amsterdam & 108 \\
10 & Washington & 101 \\
11 & Strasbourg & 100 \\
12 & Rome & 91 \\
13 & New York & 87 \\
\hline 26 & Bangkok & 51 \\
27 & Buenos Aires & 51 \\
28 & Montréal & 51 \\
\hline 36 & Toronto & 44 \\
37 & San Francisco & 39 \\
\hline 42 & & 39 \\
\hline 48 & Ottawa & 32 \\
\hline
\end{tabular}

Source: Les réunions internationales en 1990, UAI.

TABLEAU 5

Nombre de congressistes à Montréal en 1989, 1990 et 1991

\begin{tabular}{lrrr}
\hline Nombre de participants & 1989 & $\mathbf{1 9 9 0}$ & 1991 \\
\hline Palais des congrès & 299942 & 327425 & 327677 \\
Hôtels & 53611 & 65049 & 74230 \\
TOTAL: & 353553 & 392474 & 401907 \\
\hline
\end{tabular}

Source: Données foumies par l'OCTGM et le PCM.

bien informé et bien équipé. En effet, le Palais des congres dit avant tout chercher à établir le contact avec un membre de l'association internationale convoitée qui oetvre ici. Ce dernier, s'il accepte, deviendra en quelque sorte l'ambassadeur local qui se fera le porte-parole et présentera la candidature de Montréal au chapitre canadien de l'association.

Lorsque la candidature est acceptée au niveau canadien, la dernière étape consiste à ce que la société canadienne de l'association appuie la candidature de Montréal et fasse des représentations au niveau international pour finalement amener le congrès à Montréal.

Sur le marché amériçain, le travail de sollicitation est différent. Les délégations du Québec aux USA jouent un rôle important dans la diffusion de la connaissance du Québec et, de ce fait, de Montréal comme destination-congrès. Les principaux ven- deurs comme le Palais descongrès, $1^{\prime}$ Office des congrès et du tourisme du Grand Montréal, les hôteliers, couvrent aussi les foires et les bourses de voyages pour inciter les Américains à tenir leurs congrès chez-nous.

Parmi ces vendeurs, les délégués commerciaux de l'OCTGM sollicitent les grandes corporations et les associations américaines pour qu'elles tiennent à Montréal leurs réunions et congrès. De leur côté, les hôteliers qui possèdent un équipement suffisant pour recevoir réunions et/ou congrès font sensiblement le même travail que l'OCTGM pour leur établissement respectif, entre au= tres, en envoyant sur la route lèurs délégués commerciaux à la recherche de nouveaux clients. L'équipe de vente du Palais des congrès de Montréal travaille de façon similaire pour atteindre le même objectif. Certains grossistes-réceptifs font également de la sollicitation auprès de ce marché.

Toute cette sollicitation est bien sûr dirigée vers les décideurs, ceux qui choisissent le lieu où se tiendra le congrès de la corporation ou de l'association. Les organisateurs professionnels de congrès ( $\mathrm{PCO})^{(5)}$ sont souvent appelés comme conseillers pour leur venir en aide.

\section{Bilan de la demande}

En 1991, Montréal a reçu plus de 401907 participants à des congrès d'origines diverses comparativement à 392474 en 1990 et 353553 en 1989 (voir tableau 5).

Ces statistiques comprennent le nombre de participants aux activités du Palais (sauf les Montréalais) ainsi que le nombre de participants aux congrès se déroulant dans les hôtels de Montréal. Il faut savoir que les données provenant des hôtels peuvent être sous-estimées. En effet, certains hôtels ne foumissent qu une information partielle.

Outre le PCM offrant une surface de plancher de $13935 \mathrm{~m}^{2}$, au centre-ville de Montréal, plusieurs hôtels offrent aussi d'excellentes facilités pour les congrès et les réunions. Si l'on ne retient que les salles de plus de $1333 \mathrm{~m}^{2}$, on note: le Bonaventure Hilton, le Château Champlain, le Centre Sheraton, le Quatre Saisons, le Ritz Carlton, le Reine Elizabeth, le Delta Montréal, le Holiday Inn Crowne Plaza, l'Auberge des Gouverneurs le Grand, le Ramada Renaissance, le Nouvel Hôtel Montréal et le Méridien Montréal.On doit 
souligner l'ouverture récente de trois hôtels près du Palais des Congrès ( I'Intercontinental, le Holiday Inn, Jardin Sinomonde, le Furama.)

\section{Conclusion}

Comme nous l'avons vu, l'importance du marché des congrès dans l'industrie touristique n'est plus à démontrer. En effet, les retombées économiques inhêrentes à cette activité sont parfois si impressionnantes qu'il n'est pas surprenant de constater l'acharnement avec lequel se battent les villes pour s'accaparer une plus grande part de ce marché lucratif.

Sur ce plan, Montréal possède des qualités qui, entre autres, lui assurent une certaine notoriété sur le marché des congrès internationaux. Avec ses infrastructures d'accueil parmi lesquelles on retrouve les hôtels et le Palais des congrès, Montréal arrive à tirer son épingle du jeu.

A Montréal, le marché des congrès accuse une évolution grandissante et ce, malgré le récession qui persiste. En effet, durant la présente année, le marchés s'est maintenuet on a même enregistré une légère hausse du nombre de participants au Palais des congrès de Montréal. Un regard vers l'avenir nous permet d'être relativement optimiste quant àl'année 1992. En effet, les réservations du PCM à ce jour nous indique que ce dernier recevra un nombre plus élevé de congrès.

Par ailleurs, la TPS, mise en application en janvier 1991, n'affecte pas autant que ce que l'on craignait les services touristiques reliếs à l'industrie des congrès; c'est du moins le cas pour l'organisation de congrès par des organisateurs provenant de l'extérieur du Canada. Ces derniers n'ont pas à payer la TPS ou s'ils la paient, ils sont remboursés sur les services tels la location de salles, d'espaces d'exposition, l'hébergement, etc.

La région de Montréal est maintenant à l'heure des choix. Les forces de vente déployées par les différents acteurs de 1 'industrie touristique se consolident depuis quelques années grâce au climat de concertation qui s'installe de plus en plus solidement dansce secteurparticulier de l'activité touristique montréalaise. Toutefois, avec un Palais des congres qui atteint un taux de location à la limite de ses capacités, les différents niveaux de gouvernements se doivent de prendre une décision rapide sur l'agrandissement du Palais des congrès de Montrĕal au risque de perdre dans l'avenir plusieurs congrès d'importance. $f$

\section{Notes et références}

(1) Eonomics Research Associates, Metropoll III, $1989, p, 10$

[2] Hutchinson-Peid Limited, Rapport sur l'étude qualitative entreprise aupras des organisateurs de réunions aux Etats-Unis, 1986, p. 10.

(3) Ghislaine De Coninck, Les ráunlons Internationales en 1989, Union des associations internationales, Brumelles, 1909, p. 1

(4) Statistiques tirées de SOM lnc., Enquête sur les Associations quabucoisos ot las congris. Montréal, novembre 1986.

(5) Le terme PCO est une abréviation anglaise qui signifie aProfessional Comention Organizers" , II s'agit de firmes privées assistant les associations. Al'organisation du congres. On en denombre une vingtaine à Montréal. 